\title{
REVIEWS
}

\section{Pseudo-polyferrocene Coating of Double Stranded DNA with Ferrocenylnaphthalene Diimide and Its Application for Electrochemical Gene Detection}

\author{
Shigeori TAKENAKA ${ }^{\dagger}$ \\ Department of Applied Chemistry, Faculty of Engineering, Kyushu University, Fukuoka 812-8581, Japan
}

(Received March 25, 2004; Accepted April 12, 2004; Published July 15, 2004)

\begin{abstract}
Ferrocenylnaphthalene diimide derivatives (FND-1-FND-3) can intercalate into double stranded DNA (dsDNA) by a threading mode every two base pairs, resulting in the arrangement of many ferrocene molecules in the major and minor grooves of dsDNA. The pseudo-polyferrocene array templated to dsDNA thus obtained is expected to serve as a new material for nanotechnology including electrochemical gene detection. Since the pseudo-polyferrocene array formed on the electrode is analyzed electrochemically, the target gene can be detected by hybridization with a DNA probe-immobilized electrode and by the ferrocenylnaphthalene diimide binding. Ferrocenylnaphthalene diimide-based electrochemical hybridization assay established in the author's group enabled rapid and highly sensitive detection of target DNA or RNA. This system can be further extended to a field where the single nucleotide polymorphism (SNP) analysis is feasible not only for polymerase chain reaction (PCR) products but also for genomic DNA taken from human white blood cells. This system could also be used for the detection of dsDNA on the DNA chip electrochemically, especially for visualization of the DNA chips by scanning electrochemical microscopy (SECM). Electrochemical DNA chips harboring multiple electrodes on a small substrate are becoming important for gene diagnosis and related purposes because of its simplicity, high sensitivity and low cost.

[DOI 10.1295/polymj.36.503]

KEY WORDS Ferrocenylnaphthalene Diimide (FND) / Threading Intercalator / Double Stranded DNA (dsDNA) / Pseudo-polyferrocene Array / Nano Wire / Electrochemical Gene Detection / DNA Chip / Scanning Electrochemical Microscopy (SECM) /
\end{abstract}

Double stranded DNA (dsDNA) is a string-like polymer consisting of one-dimensionally stacked aromatic rings of nucleic acid bases, called as the $\pi$ way. This $\pi$-way of DNA aromatic rings is expected to act as a DNA wire for an electron transfer. The possibility of the DNA wire of a nanometer size has been discussed by many researchers. ${ }^{1}$ Another approach to a DNA nanowire was reported using the coating of dsDNA by the metals or electrochemically active polymers. For example, the coating of dsDNA with metallic silver by the reaction of silver ion with $p$-benzoquinone on the dsDNA ${ }^{2}$ or the coating of dsDNA with an electric conducting polymer was reported. ${ }^{3}$ We have successfully synthesized naphthalene diimide derivatives (FND-1-FND-3) carrying two ferrocene moieties as the electrochemically active functionalities (Scheme 1). ${ }^{4-8}$ All of these ferrocenylnaphthalene diimide derivatives bind to dsDNA by a threading intercalation mode, where their substituents are projecting out in the major and minor grooves of dsDNA. Figure 1 shows the computer modeling of a complex of FND-2 and dsDNA with the two ferrocene moieties locating in the DNA grooves. It is known that naphthalene diimide derivatives can bind to dsDNA every two base pairs by threading intercalation, the binding mode of which is in agreement with the nearest exclusion model. ${ }^{9}$ Therefore, many ferrocene moieties cover dsDNA upon binding of ferrocenyl naphthalene diimide to constitute a ferrocene-coated dsDNA. Since dsDNA was covered by redox active molecules through the threading intercalation, this will provide a new method to arrange redox molecules on the surface of dsDNA. Here, we summarize the ferrocene coating of dsDNA based on ferrocenylnaphthalene diimide derivatives and their applications for electrochemical gene detection.

\section{INTERACTION OF FND WITH DOUBLE STRANDED DNA}

Two substituents of naphthalene diimide of the ferrocenyl derivatives are located over the major and minor grooves of dsDNA, when the intercalator binds to dsDNA. ${ }^{10}$ One of the substituents needs to go through the space of the base pairs of dsDNA to form its complex with dsDNA and therefore such a kind of intercalators is called as a threading intercalator. A characteristic feature of threading intercalators is that

\footnotetext{
${ }^{\dagger}$ To whom correspondence should be addressed (E-mail: staketcm@mbox.nc.kyushu-u.ac.jp).
} 


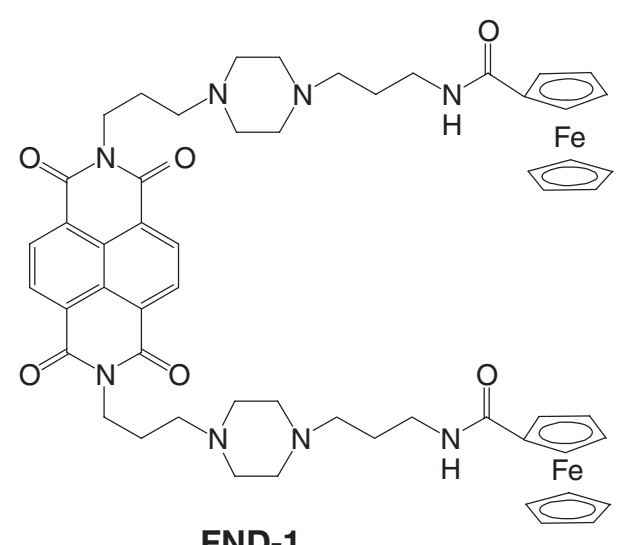

FND-1
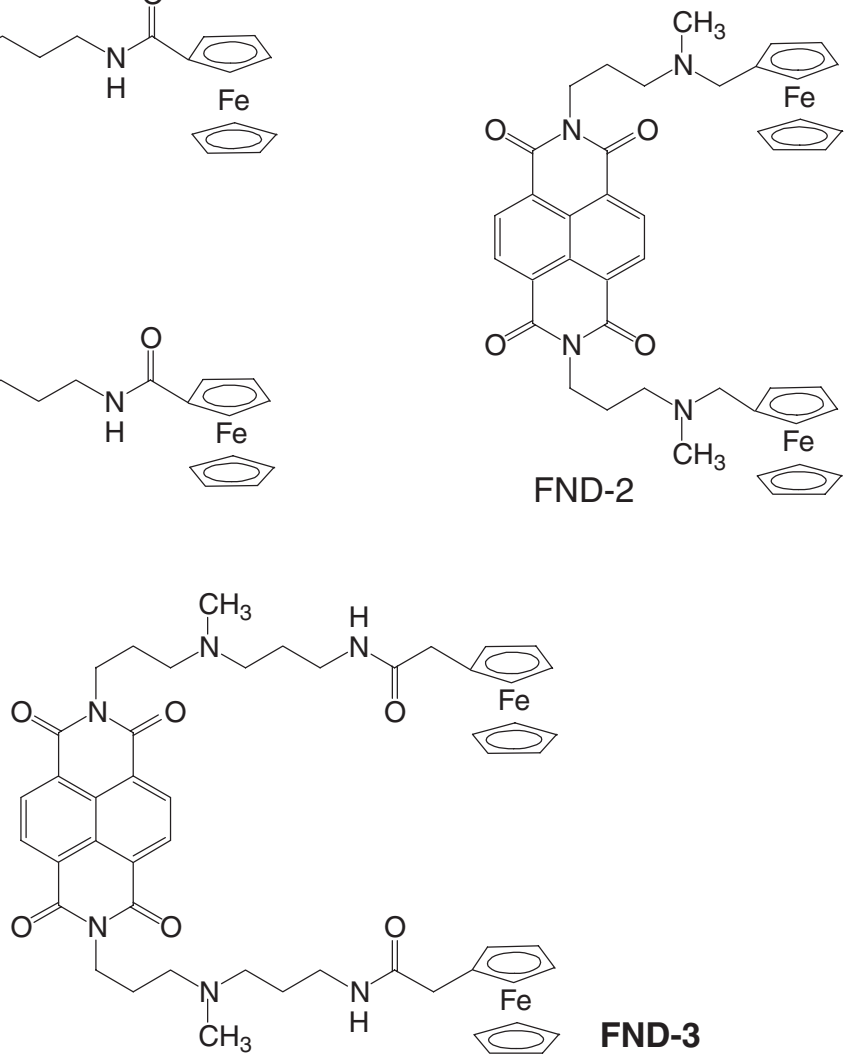

Scheme 1. Synthesized ferrocenylnaphthalene diimide derivatives, FND-1-FND-3.

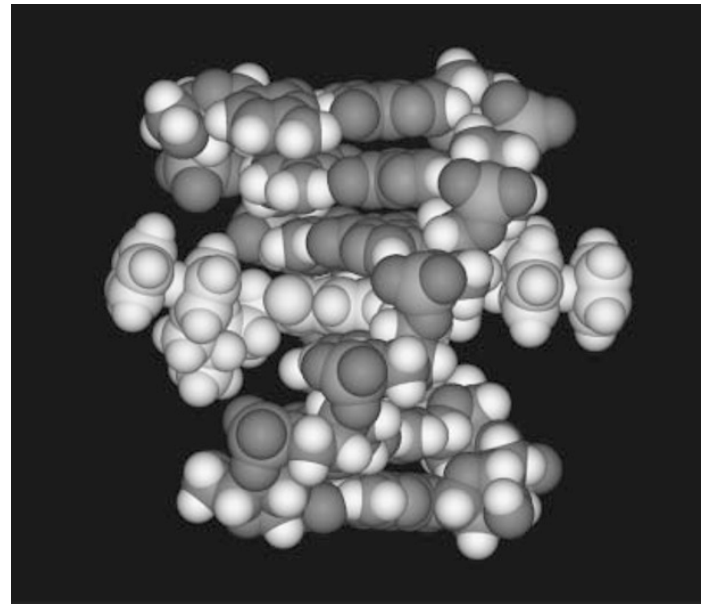

Figure 1. An Insight II molecular modeling of a complex of FND-2 with d(AAATTT $)_{2}$ as dsDNA.

two substituents projecting over the major and minor grooves of dsDNA act as an anchor to prevent the intercalator from dissociation from dsDNA and stabilize the complex with dsDNA. Nogalamycin is an antibiotic which is demonstrated to behave as a threading intercalator: its dissociation from dsDNA is very slow (dissociation rate constant is $0.001 \mathrm{~s}^{-1}$ at $0.5 \mathrm{M} \mathrm{Na}^{+}$) and this high stability of the complex with dsDNA is believed to be associated with its biological activity. ${ }^{11}$ The author's group synthesized several naphthalene diimide derivatives carrying ferrocene moieties,
FND-1-FND-3, shown in Scheme 1.4-8 They have various linker chains connecting the naphthalene diimide skeleton with ferrocene. There are different connecting patterns toward the end of the ferrocene with different redox potentials and different binding affinity for dsDNA depending upon the linker chain. FND-1 shows an absorption maximum at $383 \mathrm{~nm}$ in aqueous solution and undergoes a large hypochromic and small red shifts upon addition of dsDNA. Since a tight isosbestic point is observed during this change, it is certain that FND-1 binds to dsDNA with a single mode. If DNA is regarded as a one-dimensional lattice and FND-1 can bind to it, Scatchard isotherm can be used for analysis of FND-1 binding to DNA. Sonicated calf thymus DNA was used as dsDNA to which FND-1 binds and the data obtained were analyzed by the theoretical equation derived by McGhee and von Hippel. ${ }^{12}$ The results of this analysis reveal that FND-1 binds to DNA with every two base pairs at saturation. This binding behavior is in agreement with that of the nearest exclusion model of the classical intercalator binding. ${ }^{13}$ This complex is regarded as onedimensional polyferrocene matrixed by dsDNA where the ferrocene moieties are arranged in the major and minor grooves of dsDNA. Stopped-flow kinetic analysis yielded a dissociation rate constant, $k_{\mathrm{d}}=0.01 \mathrm{~s}^{-1}$, for FND-1 from calf thymus DNA at $0.1 \mathrm{M} \mathrm{Na}^{+} .5$ Since classical intercalators show a dissociation rate 
constant with an order of several ten $\mathrm{s}^{-1}$, FND-1 has a characteristic of a threading intercalator, i.e., slow dissociation from the dsDNA complex.

To analyze the conformational change of dsDNA by FND-1 binding, a viscometric change of dsDNA was determined in the presence of various amounts of FND-1. The viscosity of the sonicated calf thymus DNA increased upon addition of FND-1. The increase of the viscosity is a typical behavior of intercalation, where intercalation causes unwinding and extension of linear dsDNA. ${ }^{15}$ Thus, FND-1 as a threading intercalator caused a similar conformational change of dsDNA. Other ferrocenylnaphthalene diimides of FND-2 and FND-3 also showed a similar binding manner for dsDNA. This morphological change of dsDNA was observed by atomic force microscopy (AFM). First, a linear dsDNA fragment was prepared by the digestion of cyclic plasmid DNA pBR322 with restriction enzyme EcoRI. After mixing FND-2 with this DNA fragment, the mixture was placed on mica for measurement by AFM. In the AFM image in Figure 2 shows, winding strings of dsDNA were observed in the absence of FND, whereas many sticklike strings were observed in the presence of FND-2. A twining structure of two stick-like DNA strings was also observed in AFM. ${ }^{16}$

The binding behaviors of FND-1-FND-3 with single stranded DNA (ssDNA) were also analyzed analogously. In this case, a small hypochromic effect was observed in the absorption spectra of FND-1-FND-3 due to the same interactions of the intercalators with ssDNA as with dsDNA, i.e., the electrostatic interaction between the cationic sites of FND-1-FND-3 and phosphate anions of ssDNA and the stacking interaction between nucleic acid bases of ssDNA with the aromatic rings of the intercalators. These results show that all of the ferrocenylnaphthalene diimide derivatives can bind to dsDNA strongly by threading intercalation to form pseudo-polyferrocene on dsDNA.
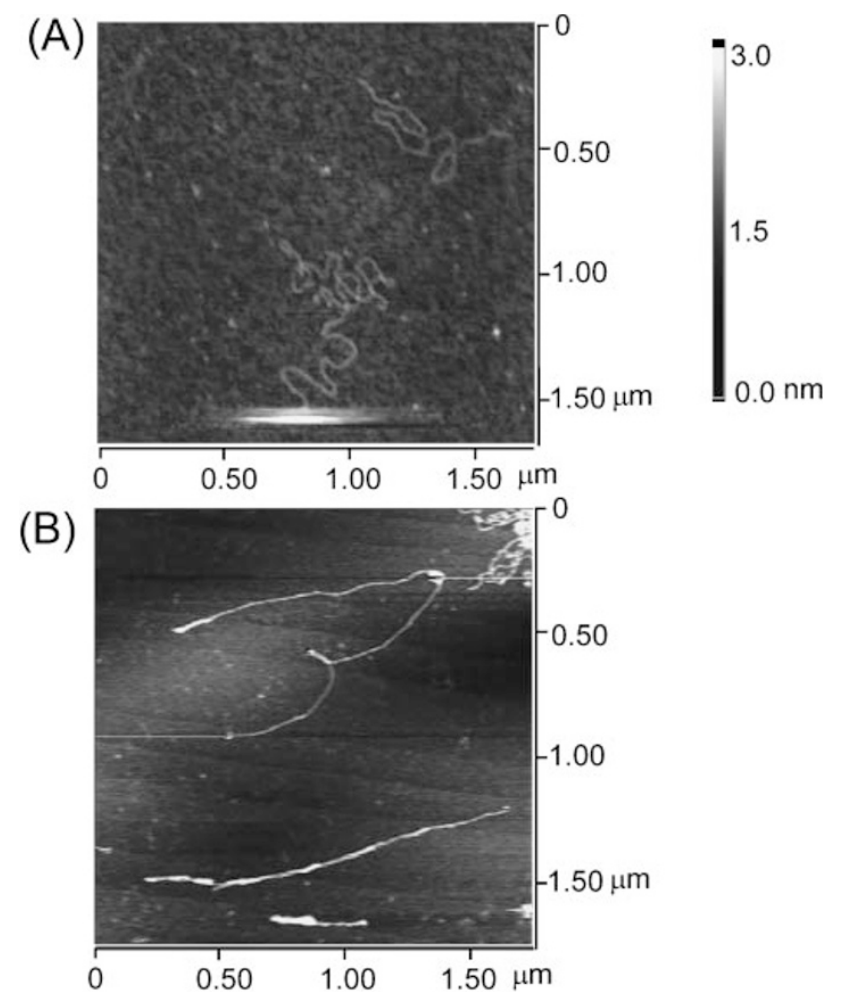

Figure 2. AFM image of a dsDNA fragment in the absence (A) and presence of FND-2 (B).

The electrochemical behavior of the pseudo-polyferrocene-coated dsDNA was evaluated by the DNA-immobilized electrode.

\section{ELECTRON TRANSFER THROUGH PSEUDO-POLYFERROCENE}

Ferrocenylnaphthalene diimide bound to dsDNA is expected to act as a DNA wire and ferrocene can mediate electron transfer to the electrode from reduced glucose oxidase. Therefore, ferrocenylnaphthalene diimide bound to dsDNA on the electrode should mediate this electron transfer reaction. The reduced glu-

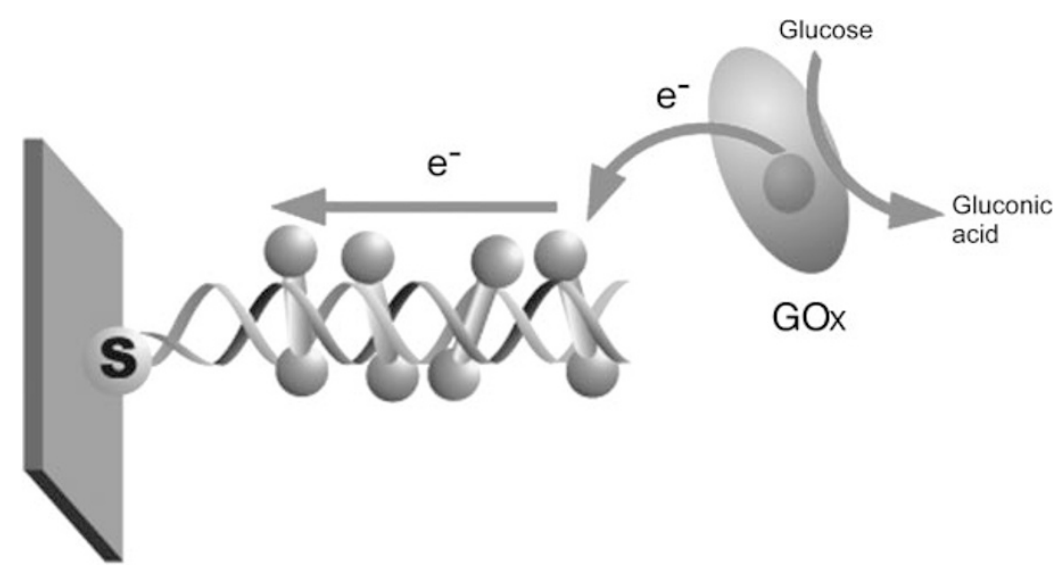

Figure 3. Cartoon of the expected electron transfer between reduced glucose oxidase (GOx) and the electrode through a pseudo-polyferrocene wire with ferrocenylnaphthalene diimide bound to the DNA duplex. 

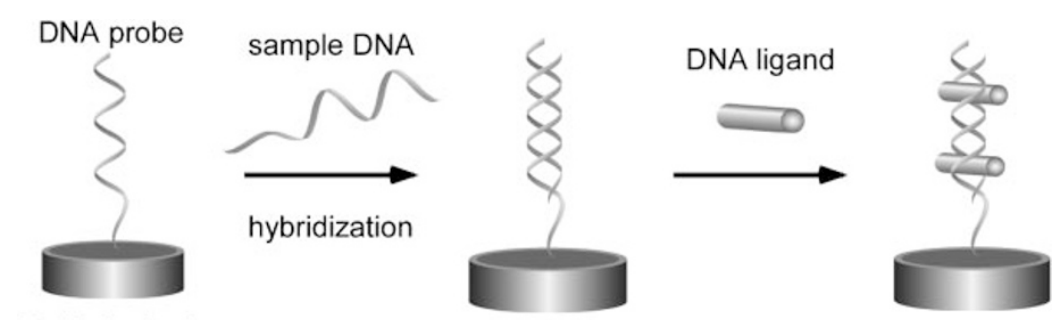

Figure 4. Principle of the electrochemical gene detection based on an electrochemical hybridization indicator.

cose oxidase was generated by its treatment with glucose. Figure 3 shows a cartoon of the expected electron transfer between reduced glucose oxidase and the electrode through the pseudo-polyferrocene wire with ferrocenylnaphthalene diimide bound to the DNA duplex.

To prove the occurrence of electron transfer, FND1 bound to dsDNA on the electrode was dipped in a solution containing reduced glucose oxidase and its cyclic voltammogram (CV) was measured. ${ }^{17}$ Control experiments were performed with ferrocenecarboxylic acid and dimethylaminomethylferrocene instead of FND-1: In the case of ferrocenecarboxylic acid, no mediated current was observed presumably due to the electrostatic repulsion between ferrocenecarboxylic acid and DNA polyanions on the electrode. On the other hand, a mediated current was observed in the cases of FND-1 and dimethylaminomethylferocene and the mediated current of FND-1 was nearly two times larger than that of dimethylaminomethylferrocene. Since cyclic voltammographic peak areas for FND-1 and dimethylaminomethylferrocene were identical to each other, their amounts concentrated on the electrode were the same. It is conceivable that larger mediated current observed for FND-1 drives from the pseudo-polyferrocene-coated DNA, as the amount of ferrocene concentrated on both electrodes is identical. However, the effect of polyferrocene was much smaller than expected, presumably because FND-1 and reduced glucose oxidase were segregated: the former as a mediator was concentrated on the dsDNA-immobilized electrode and the latter existed in the bulk solution. Therefore, the mediate reaction could occur only when the reduced glucose oxidase was diffused to the electrode. By contrast, since some fraction of dimethylaminomethylferrocene could be concentrated on the dsDNA-immobilized electrode by the electrostatic interaction and also could participate in the mediate reaction occurring in the solution, the effective mediation reaction could occur with this ferrocene. In spite of this disadvantage, the dsDNA with FND-1 bound can be expected as a DNA wire because of some trick is devised to enhance effective mediation ability of FND-1.

\section{APPLICATION FOR ELECTROCHEMICAL GENE DETECTION}

Electrochemically active ligands showing high preference for dsDNA can be used as an electrochemical hybridization indicator. ${ }^{18}$ The principle underlying this idea is shown in Figure 4. Firstly, a DNA probe (ssDNA fragment with a sequence complementary to that of the target gene) is immobilized on the electrode. This electrode is dipped in a sample DNA solution to allow hybridization to proceed. When a target DNA fragment is contained in it, dsDNA is formed with the DNA probe on the electrode. The electrochemical measurement of the electrode is made in an electrolyte containing the electrochemical hybridization indicator. The amount of the indicator concentrated on the electrode increases with an increase in the amount of dsDNA formed on the electrode and therefore the amount of target DNA can be quantitated from this electrochemical response.

As shown in Figure 4, the indicator should have the characteristics of high affinity for dsDNA without sequence specificity. So far some intercalators and groove binders are known as such an indicator. ${ }^{19,20}$ However, many intercalators or groove binders show preference for GC or AT of dsDNA, respectively. ${ }^{13}$ FND showing preference for dsDNA was expected not to show sequence preference, as FND has similar binding constants for $[p o l y(d G-d C)]_{2}$ and $[p o l y(d A-$ dT) $]_{2} .{ }^{5}$ In fact, the current observed in the application for DNA sensing was not dependent on the DNA sequence, suggesting that the main factor for the stabilization of dsDNA complex with FND-1 may be the anchoring of the substituents of FND-1 to dsDNA.

DNA detection by using FND-2 based on the principle described in Figure 4 was tested with a combination of $\mathrm{dA}_{20}$ (sample DNA) and $\mathrm{dT}_{20}$ (probe DNA). ${ }^{21}$ Thus, thiolated oligonucleotide $\mathrm{dT}_{20}$ was immobilized on a gold electrode through a thiol-gold linkage as a DNA probe and the resulting electrode was allowed to hybridize with complementary $\mathrm{dA}_{20}$ or non-complementary $\mathrm{dT}_{20}$. After dipping in an FND-2 solution, the electrode was transferred to an electrolyte without FND-2 and CV were measured. Figure 5 shows the 


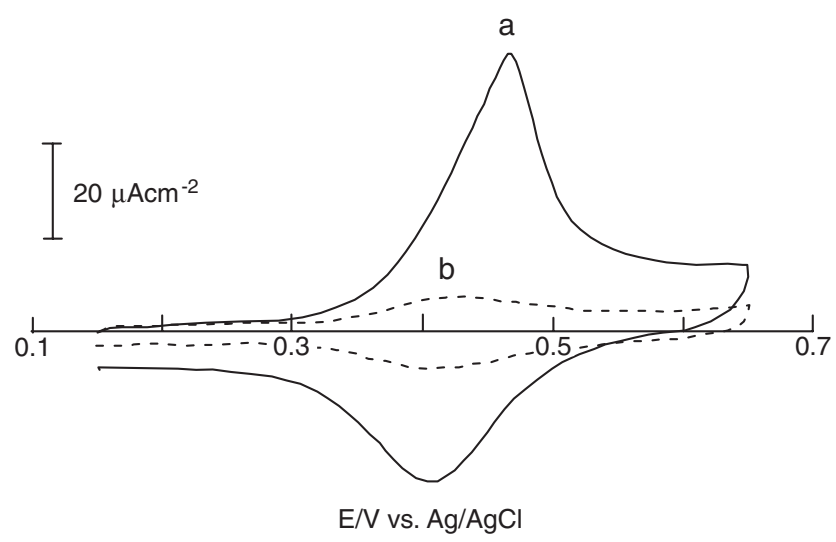

Figure 5. Cyclic voltammograms of a $\mathrm{dT}_{20}$-immobilized gold electrode in an electrolyte after hybridization with complementary $\mathrm{dA}_{20}$ (a) or non-complementary $\mathrm{dT}_{20}$, (b) followed by immersion in FND-2 solution.

$\mathrm{CV}$ of the electrode before and after hybridization with $\mathrm{dA}_{20}$. A peak current was obtained only for the complementary oligonucleotide $\mathrm{dA}_{20}$ with this electrode, whereas the signal intensity for the non-complementary DNA, $\mathrm{dT}_{20}$, was barely high above the background count. This discrimination of dsDNA from ssDNA could be attributed to the formation of a stable complex of FND-2 with dsDNA through threading intercalation.

Several kinds of DNA probe immobilization method on the electrode have been reported by many researchers. ${ }^{22}$ For example, the following procedures were reported as an effective DNA probe immobilization method: the reaction of the amino moieties of nucleic bases of a DNA probe with the activated ester of the carboxy moieties which is generated by the oxidation of a glassy carbon electrode ${ }^{23}$ or the Au-S bond formation by the reaction of thiolated DNA with the gold surface of an electrode. ${ }^{24} \mathrm{We}$ prepared a $\mathrm{dT}_{20^{-}}$ immobilized electrode by the reaction of a thiolated $\mathrm{dT}_{20}$ oligonucleotide with the pretreated gold electrode described above. Subpico mole of oligonucleotides were immobilized on average on the gold electrode with an area of $2.0 \mathrm{~mm}^{2}$. Although this method yielded a relatively constant amount of DNA probe immobilized on the gold electrode, the magnitude of the current was found variable to some extent from lot to lot in the electrochemical measurement for unknown reasons. Therefore, an index, the current shift, $\Delta i=\left(i-i_{0}\right) / i_{0} \times 100 \%$, was introduced where $i_{0}$ and $i$ refer to the current measured in an electrolyte containing FND before and after hybridization with sample DNA, respectively. Since FND binds to ssDNA mainly by electrostatic interaction between the cationic sites of FND and DNA phosphate anions, the $i_{0}$ value represents a current of the amount of DNA probe immobilized on the electrode, while the $\Delta i$ value represents a net increase of the current of the amount of dsDNA with target DNA per ssDNA of DNA probe

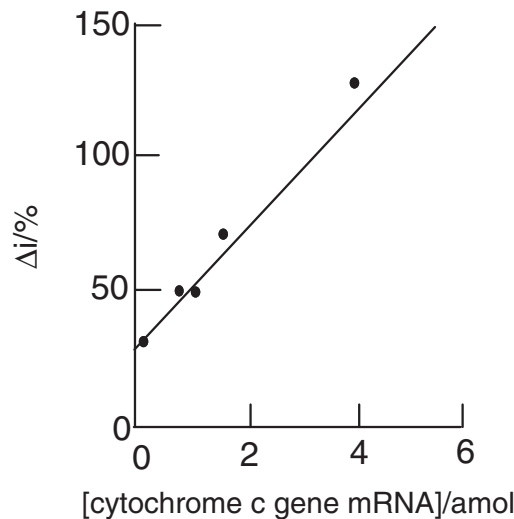

Figure 6. The $\Delta i$ values for the amount of mRNA taken from the oocyte of Xenopus. 30-meric oligonucelotide with a sequence complementary to that of the cytochrome $\mathrm{c}$ gene was used in this experiment.

immobilized on the electrode. In practice, the current obtained contains not only the current from FND absorbed on the electrode but also that from some diffusion of FND from the bulk solution. Hence, the $\Delta i$ value does not present rigorously the real amount of target DNA. Nevertheless, mRNA was detected successfully with a DNA probe-immobilized electrode as shown in Figure 6; a good correlation between the $\Delta i$ value and the amount of target mRNA was observed down to the atto mole level of target mRNA. ${ }^{25}$ In the detection of mRNA, it was allowed to hybridize with a DNA probe to form a DNA-RNA heteroduplex. The affinity of classical intercalators for the heteroduplex is lower than that for the DNA-DNA homoduplex. By contract, the threading intercalator has higher binding affinity for the heteroduplex, also suggesting that threading intercalators help to stabilize their complex with dsDNA through anchoring of their substituents. 

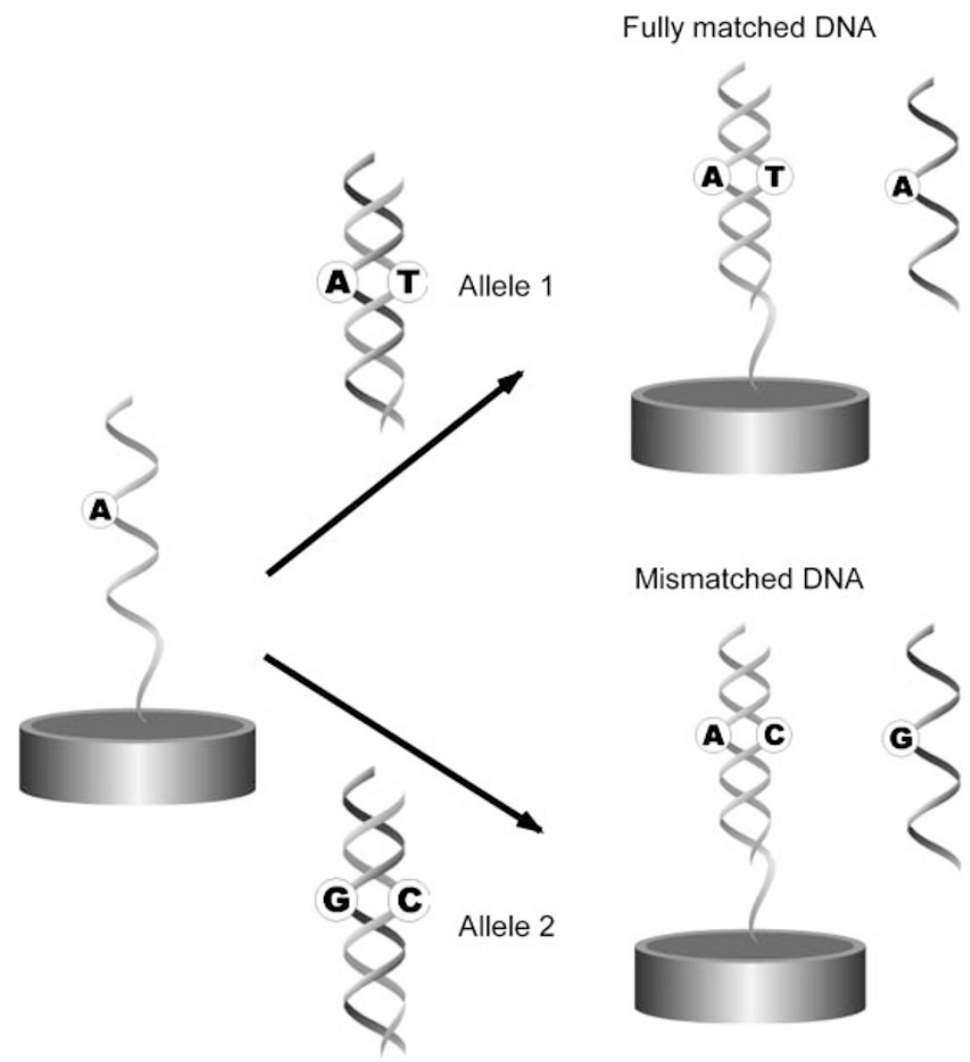

Figure 7. Principle of SNP analysis by DNA hybridization method.

\section{APPLICATION FOR SNP ANALYSIS}

SNP stands for single nucleotide polymorphism which exists every $500-1,000$ base pairs (bp) in three billion bp of human chromosomal DNA. SNP is common DNA sequence variations and strongly correlated with the individuals and hence can be used as a high density marker of the gene. ${ }^{26}$ Many SNP data are already collected and some of the SNPs are correlated with some disease or the side effect of a given drug. Therefore, the development of rapid and highly accurate SNP detection becomes a foothold of tailor-made medications. SNP detection is achieved by the DNA hybridization method whose principle is shown in Figure 7.

Let us consider here two SNP types of allele 1 and allele 2, for example, that differ in one base as shown in Figure 7. A DNA fragment representing a sequence complementary to that of allele 1 is immobilized on the electrode as a DNA probe. A DNA sample containing allele 1 or allele 2 is allowed to hybridize with the DNA probe immobilized on the electrode. A fully matched or one base mismatched DNA duplex is formed from allele 1 or allele 2, respectively. In the case of Figure 7, A/C mismatched DNA duplexes were formed from allele 2 that differ from A/T fully matched DNA duplexes in their thermal stability. In other words, the DNA duplex containing mismatched base(s) is thermally less stable because of the presence of the mismatch and tends to dissociate from the DNA probe more easily. Experimentally the discrimination of these two duplexes can be achieved by adjusting the hybridization temperature and the washing conditions such as different buffer and salt concentration after hybridization. This method is called as allele specific hybridization (ASH) and has been used for the detection of other types of gene mutation. ${ }^{27}$ As the size of probe DNA carrying a mismatch increases, the discrimination becomes more difficult. Therefore, 20-meric synthetic oligonucleotides are generally used as a DNA probe. However, the types of mismatched base pairs and neighboring base sequences also affect the magnitude of the difference and the ease of discrimination between the fully matched and mismatched DNA duplexes. From this viewpoint, SNP analysis coupled with enzymatic reaction such as Invader assay has been developed. ${ }^{28}$

Another difficulty of SNP detection arises from the existence of the same two genes in the human chromosome. As a gene type, there are a homozygote which has a mutated base on both of the two chromosomes and a heterozygote which has a matched base on one of the two chromosomes. Thus, detection of a 1:1 mixture of matched and mismatched DNA duplexes is necessary to analyze the heterozygous SNP sample. 
Firstly, we tried electrochemical SNP detection for a homozygote by using FND-1..$^{29,30}$ A twenty-meric oligonucleotide was immobilized on the gold electrode as a DNA probe and 20-meric complementary oligonucleotides carrying partially different base(s) were allowed to hybridize separately under the conditions where a DNA duplex can be formed with all of the combinations. This procedure gave mismatched DNA duplexes carrying mismatched base(s) at different sites on the duplex. The electrode in the case of fully matched or mismatched DNA duplex was used in the differential pulse voltametric (DPV) measurement in an electrolyte containing FND-1 to show that the $\Delta i$ value for the mismatched DNA duplex was smaller than that for fully matched one. This difference of $\Delta i$ values was derived from the difference in the amount of bound FND-1 per DNA duplex molecule. The amount of FND-1 bound to the DNA duplex was evaluated by quartz crystal microbalance (QCM) experiments. A twenty-meric oligonucleotide was immobilized on the gold-covered QCM plate and dipped to buffer solution. A matched or mismatched 20-meric oligonucleotide was added in this solution and a decrease of the frequency for the QCM plate was observed in both cases. An example for a complementary oligonucleotide combination is shown in Figure 8.

The amount of DNA duplex formed was estimated from the frequency decrease. When FND-1 was added to this system, the frequency of QCM decreased in proportion to FND-1 bound to the DNA duplex and the amount of FND-1 bound to DNA duplex formed on the QCM plate was calculated from the frequency decrease in each case. It was found that 9 molecules of FND-1 could bind to the 20-meric oligonucleotide duplex on average. This is reasonable in light of the nearest exclusion model and the terminal effect of the intercalation into a short DNA fragment. On the other hand, the existence of one base mismatch on

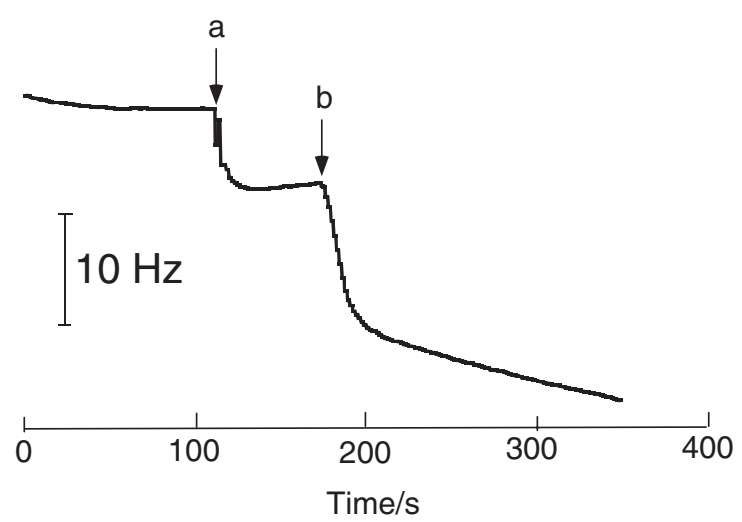

Figure 8. Frequency change for $5^{\prime}$-HS-ATT GAC CGT AAT GGG ATA GG-3' immobilized QCM plate upon successive addition of the complementary 20-meric oligonucleotide (addition point a) to form dsDNA and then FND-1 (addition point $b$ ). the 20-meric oligonucleotide duplex gave 7 molecules of bound FND-1 on average, demonstrating that FND1 could not bind to mismatched base pairs nor the base pairs neighboring mismatched one. This would result in a decrease in the $\Delta i$ values. Therefore, the existence of SNP in sample DNA can be detected from a decrease in $\Delta i$ value compared with that of the fully matched DNA duplex. This discrimination is not dependent on the thermal stability between matched and mismatched DNA duplexes, and, therefore, this discrimination method is especially effective for use as a DNA chip where many DNA probes carrying different sequences with different duplex stability are integrated in a small area of the substrate surface. However, this method is limited to the case of the DNA fragment with the same size of the DNA probe. Furthermore, the discrimination ability would decrease with an increase in their length.

To circumvent these disadvantages, temperature effect was considered combining with the mismatch discrimination. When FND bound to a DNA duplex, the resulting complex is stabilized as a result of the threading intercalation. Since the amount of bound FND is different between the fully matched and mismatched DNA duplexes, the stabilization state of the two DNA duplexes combined with FND would be different. Therefore, the difference in the thermal stability between the fully matched and mismatched DNA duplexes in the presence of FND should be larger than that in the absence of one. Proper temperature setting based on the above notion could enable the effective mismatch discrimination.

Finally, the effective one base mismatch discrimination was realized for SNP in the cancer suppression gene p53 by the electrochemical method based on FND-1 by optimizing the measurement temperature. $^{31,32}$ It is known that there are sites called hot spots on this gene that are prone to undergo mutation and the mutation results in impairment of the malignancy-suppressing ability of the p53 gene product. Electrochemical SNP analysis with a 20-meric oligonucleotide carrying the SNP site coupled with FND1 was performed for the transition to $A$ from $G$ in the codon for the amino acid at position 175, 248 or 273 and the transversion to $\mathrm{C}$ from $\mathrm{G}$ in the codon for the amino acid at position 72 . Firstly, the measuring temperature, which can discriminate between fully matched and mismatched 20-meric target oligonucleotides, was searched. Then the significantly different $\Delta i$ values were obtained at $35^{\circ} \mathrm{C}$ between fully matched and mismatched oligonucleotides. Moreover, SNP analysis for the PCR product ( $275 \mathrm{bp}$ ) of exon 4 of p53 with chromosomal DNA could be tested by this method. In this case, the detection of a heterozygote was achieved with two DNA probes corresponding 
to two alleles ( $\mathrm{G}$ or $\mathrm{C}$ type) in the codon for the amino acid at position 72 . Since the heterozygote has two types of alleles, the distinguished increase of the $\Delta i$ value should be observed. Individual PCR products from 22 unknown samples were tested and $68 \%$ were judged correctly or consistent with the sequencing result. This rate of hit is obviously lower than that for model oligonucleotides. Possible reasons for this are as follows. The $\Delta i$ value is not enough to correct the variability of individual electrodes. The size of the sample DNA is much larger than that in the oligonucleotide system (278 bp vs. 20-mer) and the hybridization efficiency of PCR product was lower than with that of the probe DNA.

A detection system with higher reproducibility was also developed by improving the immobilization method of DNA probe on the electrode. The PCR products $(350 \mathrm{bp}$ ) containing a mutated $\mathrm{G} 818 \rightarrow \mathrm{A}$ transition and G916 deletion of human lipoprotein lipase (LPL) gene were tested by this improved electrochemical DNA detection based on FND-1. ${ }^{33}$ After optimization of the detection conditions, this system was assessed on 10 unknown samples $(0.2$ pmol each of a PCR product) of two types of LPL mutation, and the genotype was correctly identified in every case. Figure 9 shows an example of the detection of a heterozygote of the LPL gene.

In the method described above, two different DNA probe-immobilized electrodes were necessary to detect the heterozygote. As a next step, we developed the detection of heterozygous SNP with one electrode. ${ }^{34}$ Since the rate of DNA duplex formation is the same with the fully matched and mismatched DNA duplexes, two allele samples containing a heterozygote was allowed to hybridize at a 1:1 ratio with a DNA probe representing one allele. When only a mismatched DNA duplex on the electrode leads to dissociation from the electrode in the measurement
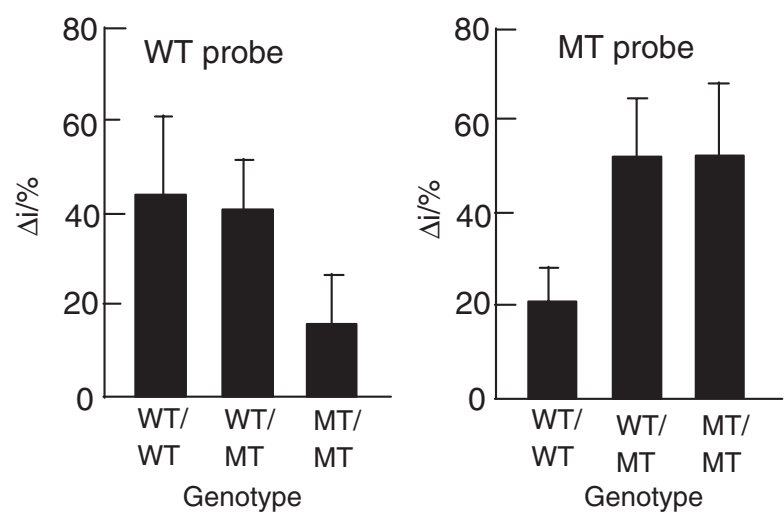

Figure 9. Electrochemical detection of a heterozygous LPL gene. The heterozygote gave rise to a significant electrochemical signal from both electrodes for wild and mutant type DNA probes. step, half of hybrid DNA of heterozygote dissociated comparing with the case of homozygote carrying the same SNP type of DNA probe. In the case of the homozygote carrying different SNP type of DNA probes, all hybrids dissociated from the electrode. Here, C- or G-type SNP is called W (wild) or M (mutant), respectively. The electrochemical measurement after this treatment should give the ratio of 2:1:0 of WT/WT, WT/MT and MT/MT for homozygote carrying same SNP type of DNA probe and heterozygote carrying different SNP type of DNA probe. A mutated $\mathrm{C} \rightarrow$ $G$ transition in a codon for Ser-447 of the human LPL gene was tested in the above method. Only DNA sample of M-type could dissociate from the electrode with the conditions using 13-meric DNA probe in $0.1 \mathrm{M} \mathrm{AcOH}-\mathrm{AcOK}(\mathrm{pH} 5.6)$ and $0.1 \mathrm{M}$ $\mathrm{KCl}$, and $0.05 \mathrm{mM} \mathrm{FND}$ at $45^{\circ} \mathrm{C}$. Under these conditions, the PCR products (231 bp) of $\mathrm{W} / \mathrm{W}, \mathrm{W} / \mathrm{M}$, and $\mathrm{M} / \mathrm{M}$ gave a 2:1:0 ratio of $\Delta i$ values by using W-type DNA probe-immobilized electrode, whereas the 0:1:2 ratio was obtained in the case of M-type DNA probeimmobilized electrode. This SNP detection for homozygote and hetrozygote was achieved directly by using chromosomal DNA digested with RNase A. Thus, ferrocenylnaphthalene diimide-based electrochemical DNA detection was successfully achieved not with PCR products, but also with genomic samples.

\section{APPLICATION FOR DNA CHIPS}

A DNA chip is now an indispensable tool for gene reseaerch where many different kinds of DNA probes are integrated on a small area and generally fluorescently labeled DNA sample is allowed to hybridize on it. After washing the DNA chip with buffer solution, many kinds of genes could be analyzed simultaneously by the fluorescence signal on the surface of the DNA chip. While, electrodes carrying many different DNA probes are integrated to give a multi DNA sensing system as an electrochemical DNA chip. This electrochemical DNA chip system is expected to constitute a more compact instrument with good cost performance. The author's group developed an electrochemical array (ECA) chip in collaboration with TUM-gene Inc. ${ }^{35}$ Toshiba Corp. (Japan) and Motolora, Inc. (USA) ${ }^{36}$ also developed electrochemical DNA chips and their readers. All of these devices have a potential to offer a relatively cheap, easy-to use and portable tool for DNA analysis and present platforms for high-throughput gene diagnosis. Recently, TUM-gene Inc. developed a simultaneous multiple mutation detection (SMMD) method coupled with enzymatic reaction. This method can detect not only a single base mutation such as SNP, but also the movement of chromosome and repeat mutation on a single 
chip that is expected as to be used a diagnostic chip.

For the first time we succeeded in electrochemical visualization of DNA chips by scanning electrochemical microscopy (SECM). ${ }^{37}$ The DNA chip was prepared by spotting a DNA solution on an aminopropylsilane-coated glass substrate with DNA microarrayer of $100 \mu \mathrm{m}$ in diameter. Sample DNA was allowed to hybridize on the DNA chip under proper conditions and measured in an electrolyte containing FND by SECM. FND is concentrated on dsDNA formed between target DNA and probe DNA on the DNA chip, enabling electrochemical visualization by SECM. Detection of a one base mutation of p53 gene was successfully achieved for the PCR product by this method. This method is potentially applicable for a conventional DNA microarray system without any special chip such as multi-electrode array. However, there are some difficulties to be overcome for keeping the DNA chip horizontally to be scanned by the probe electrode.

\section{CONCLUDING REMARKS}

The human genome project ended in April 14, 2003 with completion of the human genetic sequence. The fruit of this feat accelerates the development of the gene diagnosis including SNP analysis. The technology of the electrochemical gene sensing is a good candidate to realize this purpose. This comes from the expectation for the down sizing of instruments, rapidity of the measurement time, high sensitivity, and the extensive ability for multi-electrode system. FND bound to dsDNA with threading intercalation every two base pairs of dsDNA and formed a pseudo-polyferrocene array surface on the dsDNA. This characteristic of FND is exceedingly suitable for the electrochemical hybridization indicator in the electrochemical DNA detecting system.

As described in the review decreased here, FNDbased electrochemical DNA hybridization assay was established. Further, the SNP detection system was developed for PCR products and human genome samples. Electrochemical detection based on FND was extended to various SNP analyses and its effectiveness was presented. Detection of DNA chips generated from integrated electrodes was an example for the effective use of FND. The possibility of electrochemical visualization of conventional DNA chips was shown by using SECM. Reversible and electrochemically active new ligands having a high preference for dsDNA accelerated the research for an electroactive moleculecovered DNA wire. Further development of the electrochemical gene sensing system is highly desired for more popular use.
Acknowledgment. The author is grateful to all of his co-workers whose names appear in the papers cited in References for their enthusiasm for research. Special thanks are due to Prof. Hiroki Kondo of Kyushu Institute of Technology, Iizuka, Japan for reading the manuscript. The work described here was supported in part by Grants-in-Aid for Scientific Research from the Ministry of Education, Culture, Sports, Science and Technology, Japan (MEXT) and by New Energy and Industrial Technology Development Organization, Japan (NEDO).

\section{REFERENCES}

1. T. J. Meade, "Electron transfer reactions through the DNA double helix" in "Metal ions in biological systems," Marcel Dekker, New York, N.Y., Vol. 32, 1996, pp 453-478.

2. E. Braun, Y. Eichen, U. Slivan, and G. Ben-Yoseph, Nature, 391, 775 (1998).

3. Y. Xiao, A. B. Kharitonov, F. Patolsky, Y. Weizmann, and I. Willner, Chem. Commun, 2003, 1540.

4. S. Takenaka, Y. Uto, H. Saita, M. Yokoyama, H. Kondo, and W. D. Wilson, J. Chem. Soc., Chem. Commun., 1998, 1111.

5. S. Takenaka, K. Yamashita, M. Takagi, Y. Uto, and H. Kondo, Anal. Chem., 72, 1334 (2000).

6. S. Sato, S. Fujii, K. Yamashita, M. Takagi, H. Kondo, and S. Takenaka, J. Organomet. Chem., 637-639, 476 (2001).

7. S. Sato, K. Yamashita, M. Takagi, and S. Takenaka, Nucl. Acids Symp. Ser., 44, 171 (2000).

8. S. Sato, H. Takamiya, K. Yamashita, M. Takagi, H. Kondo, and S. Takenaka, Nucl. Acids Res. Suppl., 1, 269 (2001).

9. W. Saenger, "Principles of Nucleic Acid Structure," Springer-Verlag, New York, N.Y., 1983, pp 350-367.

10. S. Takenaka and M. Takagi, Bull. Chem. Soc. Jpn., 72, 327 (1999).

11. K. R. Fox, C. Brassett, and M. J. Waring, Biochem. Biophys. Acta, 840, 383 (1985).

12. J. D. McGhee and P. H. von Hippel, J. Mol. Biol., 86, 469 (1974).

13. W. D. Wilson, "Reversible interactions of nucleic acids with small molecules" in "Nucleic Acids in Chemistry and Biology, 2nd ed.," G. M. Blackburn and M. J. Gait, Ed., Oxford University Press, New York, N.Y., 1996, pp 329-374.

14. W. D. Wilson and F. A. Tanious, in "Molecular Aspects of Anticancer Drug-DNA Interactions," S. Neidle and M. J. Waring, Ed., Academic Press, London, Vol. 2, 1992, p 65.

15. B. M. J. Revet, M. Schmir, and J. Vinograd, Nature, 229, 10 (1971).

16. S. Sato, T. Nojima, and S. Takenaka, unpublished data.

17. S. Takenaka, Y. Uto, M. Takagi, and H. Kondo, Chem. Lett., 1998, 989.

18. S. Takenaka, Bull. Chem. Soc. Jpn., 74, 217 (2001).

19. F. Jelen, A. Erdem, and E. Palecek, J. Biomol. Struct. Dyn., 17, 1176 (2000).

20. K. Hashimoto, K. Ito, and Y. Ishimori, Anal. Chem., 66, 3830 (1994).

21. M. Komatsu, T. Nojima, and S. Takenaka, unpublished data. 
22. F. Lucarelli, G. Marrazza, A. P. F. Rurner, and M. Mascini, Biosensor Bioelectron., 19, 515 (2004).

23. K. M. Millan and S. R. Mikkelsen, Anal. Chem., 65, 2317 (1993).

24. Y. Okahata, Y. Matsunobu, K. Ijiro, M. Mukae, A. Murakami, and K. Makino, J. Am. Chem. Soc., 114, 8299 (1992).

25. S. Sato, T. Nojima, and S. Takenaka, unpublished data.

26. J. Brookes, Gene, 234, 177 (1999).

27. L. Beaudet, J. Bédard, B. Breton, R. J. Mercuri, and M. L. Budarf, Genome Res., 11, 600 (2001).

28. V. Lyamichev, A. L. Mast, J. G. Hall, J. R. Prudent, M. W. Kaiser, T. Takova, R. W. Kwiatkowski, T. J. Sander, M. de Arruda, D. A. Acro, B. P. Neri, and M. A. D. Brow, Nat. Biotechol., 17, 292 (1999).

29. K. Yamashita, M. Takagi, H. Kondo, and S. Takenka, Chem. Lett., 2000, 1038.

30. K. Yamashita, M. Takagai, H. Kondo, and S. Takenaka, Anal. Biochem., 306, 188 (2202).
31. H. Miyahara, K. Yamashita, M. Kanai, K. Uchida, M. Takagi, H. Kondo, and S. Takenaka, Talanta, 56, 829 (2002).

32. S. Takenaka, H. Miyahara, K. Yamashita, M. Takagi, and H. Kondo, Nucleosides, Nucleotides \& Nucleic Acids, 20, 1429 (2001).

33. K. Yamashita, A. Takagi, M. Takagi, H. Kondo, Y. Ikeda, and S. Takenaka, Bioconjugate Chem., 13, 1193 (2002).

34. T. Nojima, K. Yamashita, M. Takagi, A. Takagi, Y. Ikeda, H. Kondo, and S. Takenaka, Anal. Sci., 19, 79 (2003).

35. H. Miyahara, K. Yamashita, M. Takagi, H. Kondo, and S. Takenaka, Trans. IEE Jpn., 121-E, 187 (2001).

36. R. M Umek, S. W. Lin, J. Vielmetter, R. H. Terbrueggen, B. Irvine, C. J. Yu, J. F. Kayyem, H. Yowanto, G. F. Blackburn, D. H. Farkas, and Y.-P. Chen, J. Mol. Diag., 3, 74 (2001).

37. K. Yamashita, M. Takagi, K. Uchida, H. Kondo, and S. Takenaka, Analyst, 126, 1210 (2001).

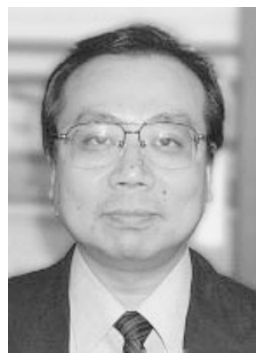

Shigeori Takenaka was born in 1959 in Fukuoka. He received his Ph D. from Kyushu University in 1988. He worked at Kyushu University as assistant professor (1987-1989) and as associate professor (1989-1991, 1996-present). He also worked at Kyushu Institute of Technology as associate professor (1991-1996). He was visiting scientist under the supervision of Professor W. David Wilson, Georgia State University of USA (1994-1995). His recent scientific interests are new analytical chemistry for biomolecules in the cell. 